\title{
COMPUTATION OF HILBERT SEQUENCE FOR COMPOSITE QUADRATIC EXTENSIONS USING DIFFERENT TYPE OF PRIMES IN $Q$
}

\author{
M. HAGHIGHI and J. MILLER \\ Department of Computer Science \\ Bradley University \\ Peoria, IL 61625 USA
}

(Received April 3, 1995 and in revised form July 3, 1995)

\begin{abstract}
First, we will give all necessary definitions and theorems. Then the definition of a Hilbert sequence by using a Galois group is introduced. Then by using the Hilbert sequence, we will build tower fields for extension $K / k$, where $K=k\left(\sqrt{d_{1}}, \sqrt{d_{2}}\right)$ and $k=Q$ for different primes in $Q$.
\end{abstract}

KEY WORDS AND PHRASES: Composite quadratic extension, Hilbert sequence 1991 AMS SUBJECT CLASSIFICATION CODES: 12A65.

\section{INTRODUCTION}

Let $K / k$ be an extension of degree $n$. We consider the tower of fields and a tower of integer rings for this extension

$$
\begin{gathered}
K \supseteq \ldots \supseteq L \ldots \supseteq k \\
O_{K} \supseteq \ldots \supseteq O_{L} \ldots \supseteq O_{k}
\end{gathered}
$$

A prime ideal $P$ in $K$ determines a prime $P_{L}$ in each field of the tower, where each $P_{L}$ is divisible by $P$. Let $p$ be a rational prime that is divisible by all these prime ideals $P_{L}$. Then we have:

$$
P_{L}=P_{k} \cap O_{L}, \quad p=P_{L} \cup Z \text {. }
$$

If the prime ideal $p$ in $k$ does not split into $n$ distinct factors of $P$ in $K$, how far can we go in terms of an intermediate field where splitting occurs? This will be answered later.

First we define what is meant by order and degree

\section{DEFINITION 1.1.}

(a) Order $P / p=e=P^{e} \mid p, p^{e+1} \chi P$

(b) Degree $P / p=f=N_{k / k} P=p^{f}$

LEMMA 1.2. Both order and degree are multiplicative

Order $P / p=\operatorname{order} P / P_{L} \cdot \operatorname{order} P_{L} / p$

Degree $P / p=$ degree $P / P_{L} \cdot$ degree $P_{L} / p$

Let us assume here that $K / k$ for $[K ; k]=n$ is a normal extension. This makes $K / L$ normal for each $L$ in the tower but not in $L / k$. Let $p$ have factors $P_{L}^{(J)}$ in $L$ for $j=1,2,3, \ldots, g$,

$$
p=\bigcap_{i=1}^{g} P_{L}^{(j) e}, N\left(P_{L}^{(\jmath)}\right)^{f}=N(p)^{f}
$$




$$
n=e . f . g \text {. }
$$

Let order $K / k P=e$ and degree $K / k P=f$. Then for $P=p$, we have order $p=$ degree $p=1$ from $k$ to $k$.

Thus from $k$ to $K$ the order has grown from 1 to $e$ and the degree has grown from 1 to $f$ and the number of factors in (1.2a) and (1.2b) has grown from 1 to $g$. We arrange the tower fields in 1.1 in such a way that will separate the growths for $K / k$ normal.

Let $K_{Z}$ be a maximal $L$ in $\{L: K \supseteq L \supseteq k\} . K_{Z}$ is called the "splitting" field of $P$ in $K / L$ and is such that

$$
\begin{aligned}
& \text { degree } P_{L} / p=1 \\
& \text { order } P_{L} / p=1
\end{aligned}
$$

Let us assume that $K_{T}$ is a maximal $L$ in $\{L: K \supseteq L \supseteq k\} . K_{T}$ is called the "inertial" field of $P$ in $K / L$ and is such that

$$
\begin{aligned}
& \text { degree } P_{L} / p=f_{L} \geq 1 \\
& \text { order } P_{L} / p=1 .
\end{aligned}
$$

This maximality process can be performed again for all $L$ such that:

$$
\begin{aligned}
& \text { degree } P_{L} / p=f_{L} \geq 1 \\
& \text { order } P_{L} / p=e_{L} \text { for }\left(e_{L}, p\right)=1 .
\end{aligned}
$$

The maximal field here is called the "first ramification" field $K_{v_{1}}$.

For this field, $F_{L}=f$ and $e_{L}$ is a part of $e$ prime to $p$. This part is called "tame ramification " If order $e$ is divisible by $p$, the ramification is called "wild." Thus we have the new tower fields for extension $K / k$ :

$$
K \supseteq \ldots \supseteq K_{v_{1}} \supseteq K_{T} \supseteq K_{Z} \supseteq k
$$

It is easier to define $1.2 \mathrm{c}$ by the Galois group methods.

DEFINITION 1.2. Let $K / k$ be a normal extension. The Hilbert sequence for an ideal $P$ in $K$ is given by the subgroups of $G=\operatorname{Gal}(K / k)$ as follows:

$$
\begin{gathered}
K \supseteq \ldots \supseteq K_{v_{1}} \supseteq K_{T} \supseteq K_{Z} \supseteq k \\
1 \subseteq \ldots \subseteq G_{v_{1}} \subseteq G_{T} \subseteq G_{Z} \subseteq G \\
k_{Z} \stackrel{G}{\leftrightarrow}\left\{u \in G: P^{u}=P \text { or } A \equiv 0=A^{u} \equiv 0 \bmod p\right\}=G_{Z} \\
k_{T} \stackrel{G}{\leftrightarrow}\left\{u \in G: P^{u} \equiv A \bmod p\right\}=\left(G_{v_{0}}\right) \\
k_{V_{r}} \stackrel{G}{\leftrightarrow}\left\{u \in G: A^{u} \equiv A \bmod p^{r+1}\right\}=G_{v_{r}}, \quad(r \geq 0) .
\end{gathered}
$$

Where $A$ is an arbitrary integer in $O_{k}$. Since $G_{Z}$ fixes $P$, then $G_{T}, G_{v}$, and so on are invariant subgroups of $G_{Z}$. Since $G_{Z}$ preserves $P$, it is one of $g$ conjugates,

$$
\left|G / G_{Z}\right|=g
$$

also, since $G_{T}$ preserves each residue class $\bmod P$,

$$
\left|G_{Z} / G_{T}\right|=\left|\left(O_{K} / P\right) /\left(O_{k} / p\right)\right|=|c(f)|=f,
$$

which refer to the cyclic Galois group of an extension of a finite field. Furthermore

$$
\left|G_{T}\right|=e \text {. }
$$


If $r=e_{0} p^{w}$, where $\left(e_{0}, p\right)=1$, then there is a cyclic quotient,

$$
\left|G_{T} / G_{v_{0}}\right|=e_{0}
$$

followed by future quotient groups of type $C(p) \times C(p) \times \ldots \times C(p)$, with

$$
G_{v_{r}} / G_{v_{r+1}}=p^{w_{r}}\left(w_{r} \geq 0, \Sigma w_{r}=w\right) .
$$

Here there is only a finite number $w_{r}>0$, indeed $p^{w} \mid n \quad$ More general details of the above can be found in [1], [2], [3], [4], [5], [6], [7]

\section{COMPUTING HILBERT SEQUENCE FOR $K=k(\sqrt{d 1}, \sqrt{d 2})$, FOR $k=Q$.}

Computing Hilbert sequence for $K=k(\sqrt{d}), k=Q$, is contained in [1, p 89]. So we process to $K \supseteq k_{\imath}=Q\left(\sqrt{d_{\imath}}\right)$ for $i=1,2,3$. Let $d_{3}=d_{1} \bullet d_{2} / t^{2}$ which means $d_{3}$ is square factor free, where $d_{2}$ is the discriminant of $k_{2}$.

Let $G=\left\{1, u_{1}, u_{2}, u_{3}\right)$, where $u_{\imath}: \sqrt{d_{\imath}} \rightarrow \sqrt{d_{\imath}}, \sqrt{d_{\jmath}} \rightarrow-\sqrt{d_{\jmath}}$ for $i \neq j$, then we have

$$
k_{2}=Q\left(\sqrt{d_{2}}\right) \stackrel{G}{\Leftrightarrow} G_{2}=\left\{1, u_{2}\right\} .
$$

Here we will build a tower of fields $K \supseteq \ldots \supseteq K_{v_{1}} \supseteq K_{T} \supseteq K_{Z} \supseteq Q$ by using the Hilbert sequence in Definition 1.2 for different types of primes $p$ in $Q$.

a Let $p=P_{1} P_{2} P_{3} P_{4}$ (unramified) where the $P_{2}$ 's are primes in $K$ for $\left(d_{1} / p\right)=\left(d_{2} / p\right)=\left(d_{3} / p\right)=1$ where:

$$
(a / p)=\left\{\begin{aligned}
1 & \text { if } x^{2}=a \bmod p \text { solvable for } x \text { integer, } a \mid p \\
-1 & \text { if } x^{2} \neq a \bmod p \text { for } x \text { integer, } a \mid p \\
0 & \text { if } a \mid p .
\end{aligned}\right.
$$

Here $f=e=1$ then $g=4$ by 1.1 . From $\left|G / G_{Z}\right|=g=4$ in (1.3e) we get that, $\left|K_{Z} / k\right|=4$ and $K_{Z}=K$ and from $\left|G_{Z} / G_{T}\right|=f=1$ in (1.3f), $\left|K_{T} / K_{Z}\right|=1$ and so $K_{T}=K$. Since $\left|G_{T}\right|=e=1$ in $(1.3 \mathrm{~g})$, and from $\left|G_{T} / G_{v_{0}}\right|=e_{0}=1$ in (1.3h) and (1.3i) for $r=0,1,2,3$ then $\left|G_{v_{r}} / G_{v_{r+1}}\right|=\left|K_{v_{r+1}} / K_{v_{r}}\right|=1$

$$
K_{v_{1}}=K_{v_{2}}=K_{v_{3}}=K_{v_{4}}=K .
$$

Thus, we have the following field tower for $K / k$ :

$$
\begin{gathered}
k=Q \subseteq K_{Z} \subseteq K_{T} \subseteq K_{v_{1}} \subseteq K_{v_{2}} \subseteq K_{v_{3}} \subseteq K_{v_{4}} \subseteq K \\
Q \subseteq K=K=K=K=K=K=K=K .
\end{gathered}
$$

b Let $p=P_{1} P_{2}$ (unramified) for $-\left(d_{1} / p\right)=-\left(d_{2} / p\right)=\left(d_{3} / p\right)=1$. Here $e_{1}=e_{2}=1$, $f_{1}=f_{2}=2$ and $g=2$. Again from $\left|G / G_{Z}\right|=g=2$, we have: $\left|K_{Z} / k\right|=2$ and by (1.3b) $K_{Z}=k_{3}=Q\left(\sqrt{d_{3}}\right)$. From $\left|G_{Z} / G_{T}\right|=f=1$ then $\left|K_{Z} / K_{T}\right|=2$ and then $K_{T}=K$ Using the same proof as above: $K_{v_{1}}=K_{v_{2}}=K_{v_{3}}=K_{v_{4}}=K$. This produces the following tower fields for $K / k$ :

$$
\begin{gathered}
k=Q \subseteq K_{Z} \subseteq K_{T} \subseteq K_{v_{1}} \subseteq K_{v_{2}} \subseteq K_{v_{3}} \subseteq K_{v_{4}} \subseteq K \\
Q \subseteq k_{3} \subseteq K=K=K=K=K=K .
\end{gathered}
$$

c $p=P_{1}^{2} \cdot P_{2}^{2}$, where $p$ is odd and $p\left|d_{1}, p\right| d_{2}, p \mid d_{3}$ and $\left(d_{3} / p\right)=1$. Here $e_{1}=e_{2}=2$ and $f_{1}=f_{2}=1$ and so $g=2$ Since again $\left|G / G_{Z}\right|=g=2$ then $\left|K_{Z} / k\right|=2$ and by (1 $3 \mathrm{~b}$ ) $K_{Z}=k_{3}=Q\left(\sqrt{d_{3}}\right)$. From $\left|G_{Z} / G_{T}\right|=f=1$ then $K_{T}=K_{Z}=k_{3}=Q\left(\sqrt{d_{3}}\right) \quad$ From $\left|G_{T}\right|=e=2=e_{0} \cdot p^{w}=1 \cdot 2^{1}$ then by (1.3i) $\left|G_{v_{r}} / G_{v_{r+1}}\right|=p^{w} r=2^{1}$ and from here for $r=0$ : 
$\left|G_{r_{0}} / G_{v_{1}}\right|=\left|K_{v_{1}} / K_{T}\right|=2$ and thus $K_{v_{1}}=K$ and also $\quad K_{v_{2}}=K_{v_{3}}=K_{v_{4}}=K$, because $\left|G_{v_{r}} / G_{v_{r+1}}\right|=\left|K_{v_{r+1}} / k_{v_{r}}\right|=2^{0}=1$ which produces the following tower fields for $K / k$

$$
\begin{gathered}
k=Q \subseteq k_{Z} \subseteq k_{T} \subseteq k_{v_{1}} \subseteq k_{v_{2}} \subseteq k_{v_{3}} \subseteq k_{v_{4}} \subseteq K \\
Q \subseteq K_{3}=k_{3} \subseteq K=K=K=K=K .
\end{gathered}
$$

d. $\quad p=P_{1}^{2}$ for $p$ odd, $p\left|d_{1}, p\right| d_{2}, p \mid d_{3},(d 3 / p)=-1$ with the same proof as above, the following tower fields are produced.

$$
K_{Z}=Q, K_{T}=k_{3} \text {, and } K_{v_{1}}=K_{v_{2}}=K_{v_{3}}=K_{v_{4}}=K \text {. }
$$

e. $P=p_{1}^{2} p_{2}^{2}$, and $d_{1} \equiv d_{2} \equiv 1^{2}(\bmod 16), d_{3} \equiv 1(\bmod 8)$ produces the tower

$$
\begin{gathered}
k=Q \subseteq k_{Z} \subseteq k_{T} \subseteq K_{v_{1}} \subseteq K_{v_{2}} \subseteq K_{v_{3}} \subseteq K_{v_{4}} \subseteq K \\
Q=Q \subseteq k_{3} \subseteq K=K=K=K=K .
\end{gathered}
$$

f. $p=p_{1}^{2}$ for $d_{1} \equiv d_{2} \equiv 12(\bmod 16), d_{3} \equiv 5(\bmod 8)$. Here $e=2$ and $g=1$ then $f=2$. From $\left|G / G_{Z}\right|=g=\left|K_{Z} / Q\right|=1, K_{Z}=Q$ and by $\left|G_{Z} / G_{T}\right|=f=\left|K_{T} / K_{Z}\right|=2, K_{T}$ is a quadratic extension over $Q$, then by (1.3c) $K_{T}=k_{3}, e=2=e^{0} \cdot p^{w}=1.2^{w}$ and $\left|G_{v_{r}} / G_{v_{r+1}}\right|=2^{w_{r}}$ where $\Sigma$ $w_{r}=w$ and $w_{r} \geq 0 . \quad$ From $\left|G_{v_{0}} / G_{v_{1}}\right|=\left|K_{v_{1}} / K_{T}\right|=2^{0}=1, K_{v_{1}}=k_{3} . \quad\left|G_{v_{2}} / G_{v_{1}}\right|=2^{1}$ $=\left|K_{v_{2}} / G_{v_{1}}\right|=2$, then $K_{v_{2}}=K$, and with some proof $K_{v_{2}}=K_{v_{3}}=K_{v_{4}}=K$ producing

$$
\begin{gathered}
k=Q \subseteq K_{Z} \subseteq K_{T} \subseteq K_{v_{1}} \subseteq K_{v_{2}} \subseteq K_{v_{3}} \subseteq K_{v_{4}} \subseteq K \\
Q=Q \subseteq k_{3}=k_{3} \subseteq K=K=K=K .
\end{gathered}
$$

g. $\quad p=p_{1}^{2} p_{2}^{2}$ for $d_{1} \equiv d_{2} \equiv 8(\bmod 16), d_{3} \equiv 1(\bmod 8)$ has the same tower fields as $e$.

h. $\quad p=p_{1}^{2}$, for $d_{1} \equiv d_{2} \equiv 8(\bmod 16), d_{3} \equiv 5(\bmod 8)$ also has the same Hilbert sequence as $f$.

i. $\quad p=p_{1}^{4}$ for $d_{1} \equiv d_{2} \equiv 8(\bmod 16), d_{3} \equiv 12(\bmod 8)$ has the following tower fields

$$
\begin{gathered}
k=Q \subseteq k_{Z} \subseteq K_{T} \subseteq K_{v_{1}} \subseteq K_{v_{2}} \subseteq K_{v_{3}} \subseteq K_{v_{4}} \subseteq K \\
Q=Q=Q=Q \subseteq k_{3}=k_{3} \subseteq K=K .
\end{gathered}
$$

We showed in the above cases, if the prime ideal $p$ of $k$ does not split into $n$ distinct prime factors of $K$, how we can build intermediate fields $K_{Z}, K_{T}, K_{v_{0}}, \ldots$ where splitting of prime $p$ occurs.

ACKNOWLEDGMENT. Thanks to the referees for many valuable suggestions for improving the content of the paper.

\section{REFERENCES}

[1] COHN, H., Introduction to the Construction of Class Fields, Cambridge University Press, 1985

[2] HILBERT, D., Die theorie der algebraischen Zahlkörper, Jahrsber. Deutsch. Math. 4 (1897), 173546.

[3] HILBERT, D., Über die theorie des relativ quadratischen Zahlkörpers, Math. Ann. 51 (1899), 1-27.

[4] HECKE, E., Vorlesungenüber die theorie der algebraischen, Zahlem. Leipzig: Teubner, 1923.

[5] HASSE, H., Klassenkörper theorie, Original Lecture Notes, Marbug, 1933.

[6] HAGHIGHI, M., Relative Integral Bases for Algebraic Number Fields, Ph.D. Dissertation, Dept of Mathematics, CUNY, 1982.

[7] McCARTHY, P. J., Algebraic Extensions of Fields, Blaisedell Publishing Company, 1966. 


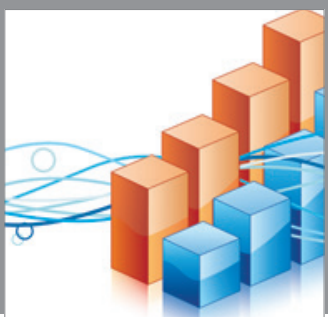

Advances in

Operations Research

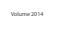

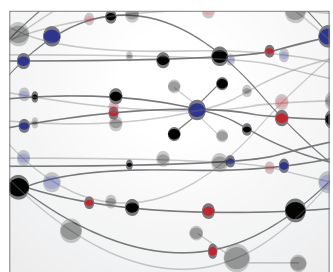

\section{The Scientific} World Journal
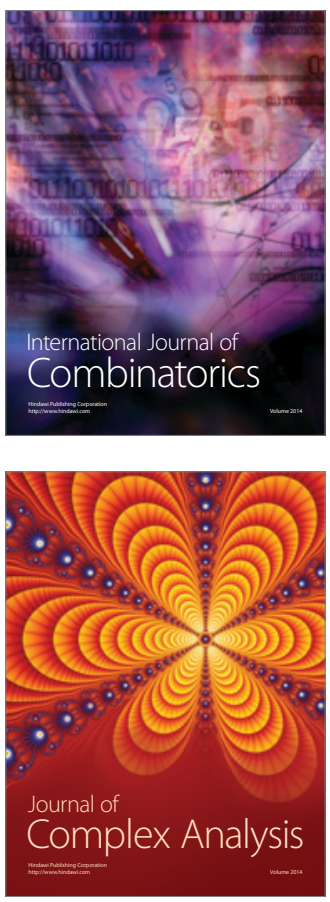

International Journal of

Mathematics and

Mathematical

Sciences
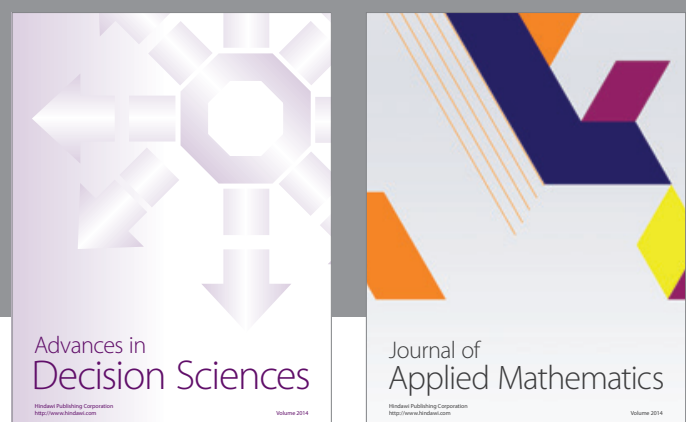

Journal of

Applied Mathematics
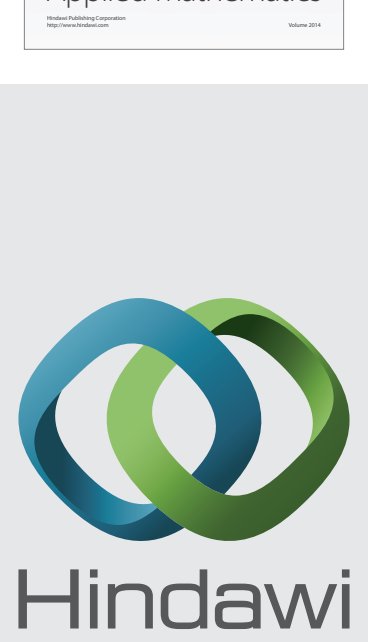

Submit your manuscripts at http://www.hindawi.com
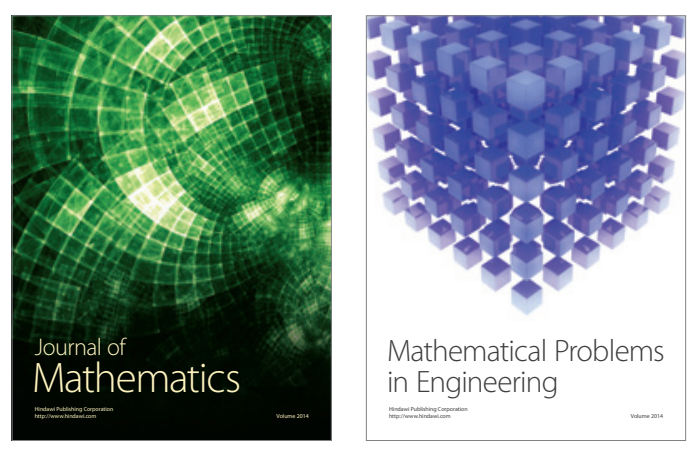

Mathematical Problems in Engineering
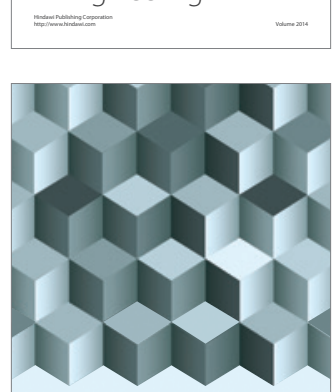

Journal of

Function Spaces
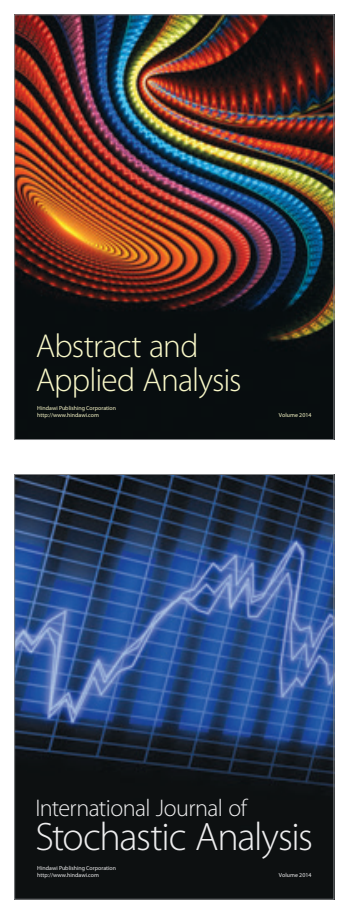

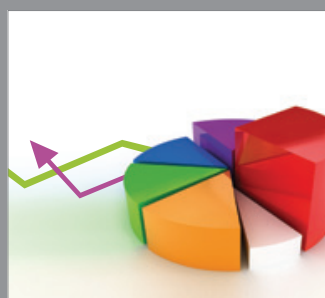

ournal of

Probability and Statistics

Promensencen
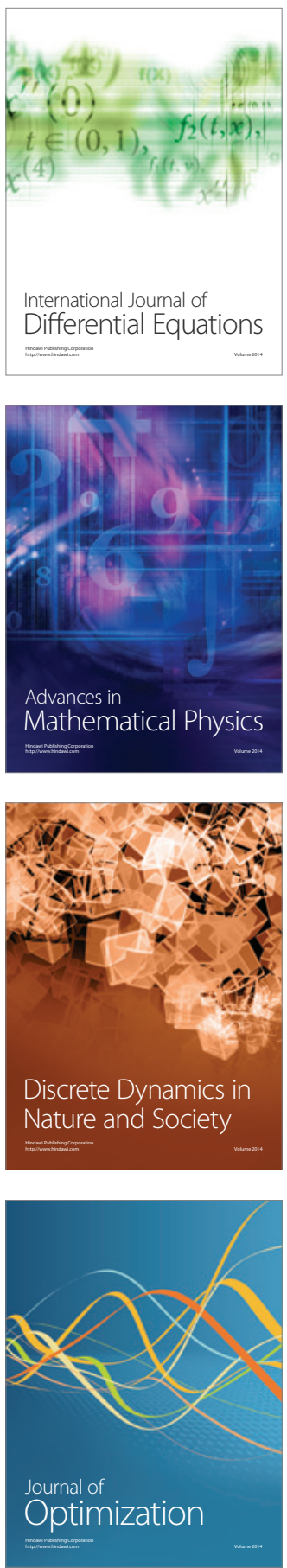